\title{
On the New Physical Biology
}

\section{George Czerlinski*}

Department of Biology, Western Washington University, Bellingham, WA 98225, USA

The concept of physical biology has been used before, particularly in the context of cells. There is now a journal with that name with a focus on physical oncology. The term physical biology covers a wide range of scales, incorporating changes in molecular biology, cellular biology and systems biology.

"Vortex" in this journal's name refers to electric charges moving in a loop and producing a magnetic field. These charges may be a single electron or a large number of electrons (charge clusters). There may even be groups of rotating charges moving in a loop, producing circulating magnetic fields. Such circulating charges could appear over a wide range of scales, from nanometers to many meters. A vortex in the natural environment is thus of interest to this journal.

The rotational speed of the electric change depends on the resistance of the environment in which the charge moves. In special cases this resistance approaches zero and we have superconducting systems, mostly resulting from quantum physical events. Thus, the new physical biology includes aspects of quantum physics. However, nuclear spin and electron spin are not considered subjects of this journal.

To explain the movement of small polymers of water in a magnetic field, the bound radical electron (in singlet excited state) needs to rotate as in a superconductor. Superconducting electronic activity also permits (near) loss-less reflection of electromagnetic waves. Ultrarapid switching between the energies of the excited state and the wave state result in much longer life of the energy quantum compared to that leading to fluorescence emission (around $10^{9} \mathrm{~s}$ versus $10^{-9} \mathrm{~s}$ for the latter in an otherwise undisturbed system). Such vast extension in lifetime increases the role which excited states may place in living systems.

The above switching process involves a minimum of two polymers, which we call nanodomains. In nature, 1000 or more nanodomains cooperate coherently, switching between states, forming coherence domains. The fast switching in coherence domains protects the stored energy from dissipating into the environment, and their number overcomes the noise present in all interactions. Such properties facilitate participation in changes at the molecular, cellular and systems level. Measurement of the energies involved (as electromagnetic frequencies or characteristic spectra) allows us to investigate the correlation between stored electromagnetic energies and states of health. The implications for medical treatment could be far-reaching. Possible benefits extend beyond human health to include animals and plants. Preliminary data on selected crops indicate that both quality and quantity of crops are increased significantly by the addition of systems rich in coherence domains.

Our own studies focus on mechanistic changes due to long-lived excited states and between molecules, cells and systems with time [1]. A possible mechanism for "gating" information into and out of coherence domain is discussed by Meyl [2]. We have preliminary data for the cellular level. Data for biological systems will follow. We have used classical kinetics in our simulations, but for association to and dissociation from coherence domains a different approach may be beneficial.

\section{Acknowledgement}

The author thanks Tjalling Ypma, D.Phil., and Robert M. Davidson, M.D. Ph.D., for their help in providing clarity and breadth of research endeavors.

\section{References}

1. Czerlinski G, Ypma T (2013) Action of excited state molecular networks. J Vortex Sci. \& Techn. in press 1: 1-8.

2. Meyl K (2012) DNA and Cell Resonance Magnetic Waves Enable Cell Communication. DNA and Cell Biology 31: 422-426.
*Corresponding author: George Czerlinski, Department of Biology, Western Washington University, Bellingham, WA 98225,USA, Tel: 928-284-9137; Fax: 480445-9773; E-mail: gczerlinski@gmail.com

Received September 29, 2013; Accepted September 29, 2013; Published September 29, 2013

Citation: Czerlinski G (2013) On the New Physical Biology. Fluid Mech Open Acc 1: e106. doi: 10.4172/2090-8369.1000e106

Copyright: (C) 2013 Czerlinski G. This is an open-access article distributed under the terms of the Creative Commons Attribution License, which permits unrestricted use, distribution, and reproduction in any medium, provided the original author and source are credited. 